\title{
Frequency Selection of Sine Wave for Dynamic ADC Test
}

\author{
M. Komárek, J. Roztočil \\ Faculty of Electrical Engineering, Czech Technical University in Prague, Technická 2, CZ-16627 Prague 6, Czech Republic \\ E-mail: milan.komarek@gmail.com,roztocil@fel.cvut.cz \\ The paper deals with determination of an optimum frequency for the time domain and frequency domain ADC testing. Proposed \\ algorithm for selection of test signal frequency fulfills two common requirements. The first requirement is to get maximum \\ quantity of distinct phases of the sampled values which are uniformly distributed between 0 and $2 \pi$; the second one is to avoid \\ overlapping of higher harmonic components aliased in the first Nyquist zone. The algorithm was verified using MATLAB \\ simulations and practical measurements.
}

Keywords: ADC testing, sine wave testing, frequency selection, windowed DFT, aliasing

\section{INTRODUCTION}

$\mathrm{T}$ HE PRECISE SELECTION of the input and sampling frequencies, and selection of the record length are very important for dynamic testing $\mathrm{AD}$ converters and modules by sine wave signal.

IEEE Std 1241-2000 [1] and Std 1057-2007 [2] provide standardization in the ADC testing. Selection of optimal input testing signal frequency under these standards is resolved separately for applied deterministic testing methods. To compare results between each test method, it is necessary to use identical test conditions inclusive of identical input testing signal frequency. That is why an algorithm of input testing signal frequency selection was developed to allow both the sine fit and the DFT methods to be applied.

\section{TIME DOMAIN CONSIDERATION}

In order to determine an ADC's performance, it is necessary to secure maximum quantity of distinct input phases that are sampled by ADC in whole record, and if possible to get at least one sample for every ADC code.

The well-known relation [1] Eq. (45) between the number of testing signal periods and the number of sampling periods can be used to secure maximum quantity of distinct input phases. It is required to find an integer $J$ which is relatively prime to the number of samples in the record $M$ (usually $M$ is a power of two), then optimum frequency is given by

$$
f_{\text {opt } 1}=\frac{J}{M} f_{s}
$$

Algorithm for calculating a near-optimum frequency $f_{\text {nearopt }}$ which is close to any desired frequency is described in [1] (Eq. (47), (48)) in three steps. Step one is finding an integer $r$, such that the desired frequency is approximately $f_{s} / r ; r \in$ $N$. Step two is calculating the number of full cycles $D$ that can be recorded at the frequency $f_{s} / r$ :

$$
D=\left\lfloor\frac{M}{r}\right\rfloor
$$

and the final step is calculating the near-optimum frequency by

$$
f_{\text {nearopt }}=\frac{D f_{s}}{r D-1}
$$

where $\quad r$ is an integer number,

$D$ is the number of acquired full periods of input sine wave with frequency $f_{s} / r$,

$f_{s}$ is the sampling frequency,

\lfloor\rfloor rounds the $X$ to the nearest integer towards zero.

This algorithm is offered for three or four-parameter sine fit method. Using input signal with frequency $f_{\text {nearopt }}$ guarantees $(r D-1)$ distinct sampled phases uniformly distributed between 0 and $2 \pi$ radians. It means that the number of sampled phases is less than but almost equal to the number of samples $M$.

The frequency $f_{\text {nearopt }}$ achieved by the described algorithm is very close to the frequency that is an integer fraction $f_{s} / r$ of sampling frequency. It may not be really important in the case of the sine fit test method but there could be a serious problem in the case of the DFT test method.

\section{FREQUENCY DOMAIN CONSIDERATION}

The spectrum of acquired data record contains fundamental and higher harmonics, spurious and intermodulation components.

Because coherent sampling cannot be guaranteed for all components present in the spectrum of the tested ADC, the windowed DFT method is assumed in the following text. The reasonable assumption that the number of samples is equal to the power of two is usually required for FFT computation.

The harmonic components are mostly dominant in the record and levels of these components have to be measured. These components lie at integer multiple of fundamental frequency and they are aliased to the first Nyquist zone (basic frequency spectrum between 0 and $f_{s} / 2$ ) provided that their frequencies are higher than $f_{s} / 2$. The fundamental frequency $f_{\text {nearopt }}$ is close to an integer fraction of sampling frequency $f_{s} / r$ and the frequencies of higher harmonic 
components are integer multiples of fundamental frequency. In that case, the aliased harmonic components can be mutually overlapped. This effect could be enlarged by the leakage or by the window main lobe size if the window function is applied to DFT as it is demonstrated in the following example.

The test signal frequency is selected to obtain the maximum of distinct phases according to the algorithm described in IEEE 1241 Std. The test signal with frequency (3) $f_{\text {nearopt }} \approx 666687.0173 \mathrm{~Hz}$ is acquired with a sampling rate of $10 \mathrm{MSa} / \mathrm{s}$ to get data record of 32768 samples $(r=$ $15, D=2184)$. There are $(r D-1)=32759$ samples which have distinct input phases uniformly distributed between 0 and $2 \pi$.

Let's assume 20 higher harmonic components nonoptimally aligned in the first Nyquist zone that are mutually overlapped with the DC component, and seven harmonic components in the first Nyquist zone. The frequency spectrum of sine wave test signal with the input test frequency given by (1) is shown in Fig.1. The measured frequency spectrum acquired by using a 14-bit digitizer can be seen in Fig.2.

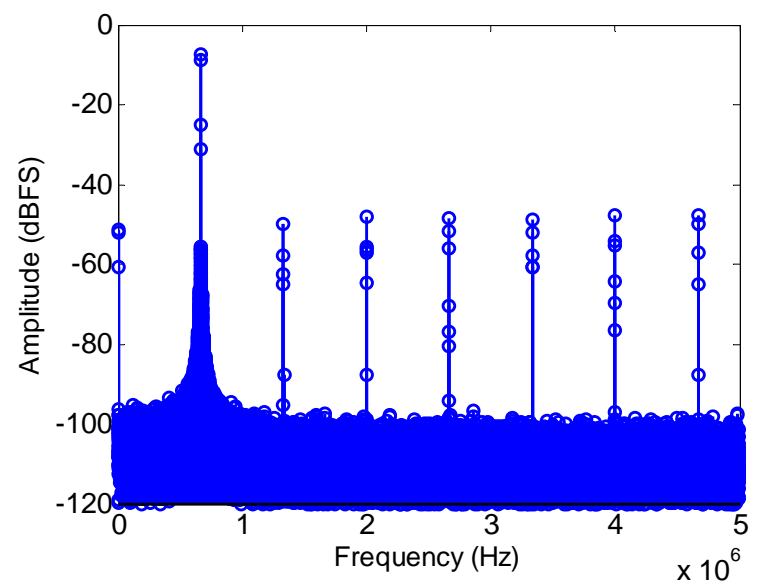

Fig.1. Simulated frequency spectrum with 20 mutually overlapped higher harmonic components $f_{\text {nearopt }} \approx 666687.0173 \mathrm{~Hz}$

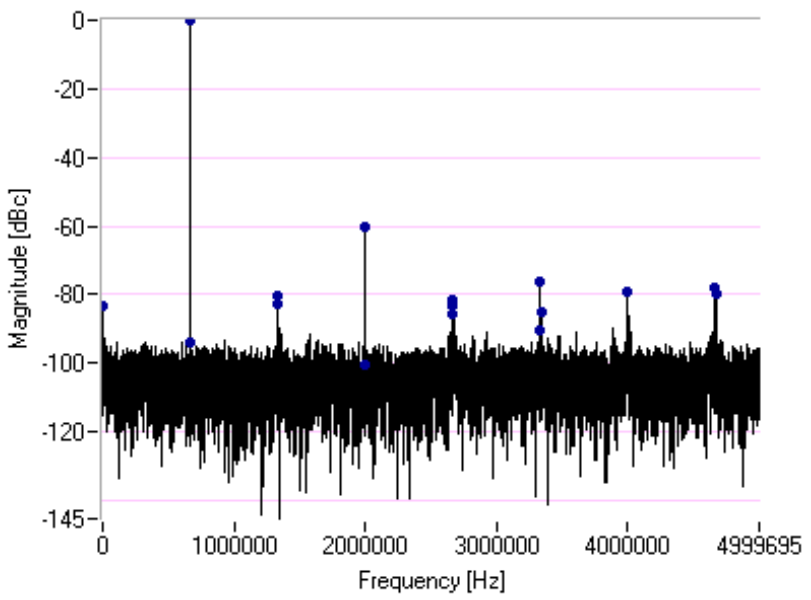

Fig.2. Really measured frequency spectrum with 20 mutually overlapped higher harmonic components $f_{\text {nearopt }} \approx 666687.0 \mathrm{~Hz}$
The sine wave signal with frequency $f_{\text {nearopt }}$ computed by (3) can be applied successfully to the sine fit and histogram test method but the level of mutually overlapped higher harmonic components can be hardly estimated from the output ADC spectrum due to the energy spread on several spectrum bins by the window used (see Fig.3). The frequency spectrum of such signal computed by DFT has non-optimally distributed higher harmonic components which are overlapped in the first Nyquist zone. For these reasons, the signal with frequency $f_{\text {nearopt }}$ is not suitable for the DFT test method.

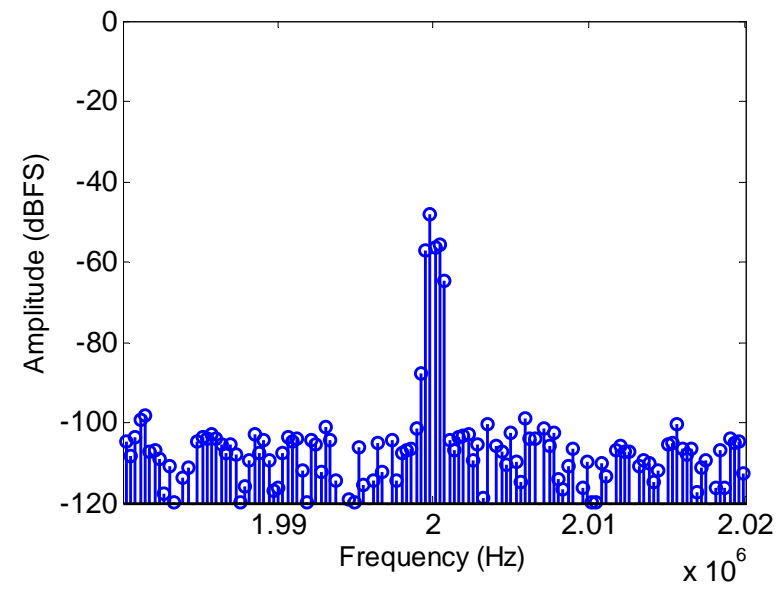

Fig.3. Zoom of windowed frequency spectrum around the $3^{\text {rd }}$ harmonic component

To avoid the overlapping of higher harmonic components, another approach has to be proposed. The frequencies of higher harmonic components can exceed $f_{s} / 2$. In that case, the frequencies of higher harmonics are aliased. They are present in the first Nyquist zone. Position of each aliased harmonic component represented by DFT bin in the frequency domain is given by

$$
f_{a}=\left|f_{\text {in }}-f_{s} \cdot\left\|\frac{f_{\text {in }}}{f_{s}}\right\|\right|
$$

where $f_{a}$ is aliased frequency,

$$
f_{i n} \text { is frequency of input signal }>\frac{f_{s}}{2} \text {, }
$$

|| || rounds to nearest integer

To prevent overlapping of higher harmonic components, it is necessary to select the input frequency $f_{\text {in }}$ in such way that all harmonics are uniformly distributed in the first Nyquist zone. This requirement is fulfilled if the input test signal frequency is in accord with the following equations

$$
k=\left\lfloor\frac{h+1}{b}\right\rfloor
$$

$$
f_{\text {opt } 2}=f_{s} \frac{k}{2(h+1)}
$$


where $\quad k \in N$ (must be odd number),

$h$ is the number of significant harmonic components in the spectrum,

$b$ is the estimated number of non aliased harmonic components in the basic spectrum.

However, there are two necessary conditions that have to be fulfilled. The first one is that $(h+1)$, which represents the number of intervals, is relatively prime to $k$. The second one is that $k$, which represents a position of fundamental harmonic component as a multiple of interval $\frac{f_{s}}{2(h+1)}$, is

an odd number. If any of the mentioned conditions are not met, the algorithm fails. The simple solution preventing the algorithm fail is based on increasing of the significant harmonic components in the frequency spectrum until both of the mentioned conditions are met.

Let us assume that the frequency spectrum of tested ADC contains up to 21 harmonic components including fundamental ones, $h=21$, and that seven harmonic components, $b=7$, are required to lie in the first Nyquist zone $\left(0\right.$ to $\left.f_{s} / 2\right)$. The last requirement limits the frequency of input test signal in the range from $\frac{f_{s}}{2 \cdot 8}=625 \mathrm{kHz}$ to

$$
\frac{f_{s}}{2 \cdot 7}=714.285 \mathrm{kHz}
$$

The test signal with frequency $f_{o p t 2} \approx 681818.1818 \mathrm{~Hz}$ is acquired with a sampling rate of $10 \mathrm{MS} / \mathrm{s}$ to get 32768 samples as shown in Fig.4, where the test signal frequency is selected in accord to prevent overlapping of higher harmonic components in the frequency spectrum.

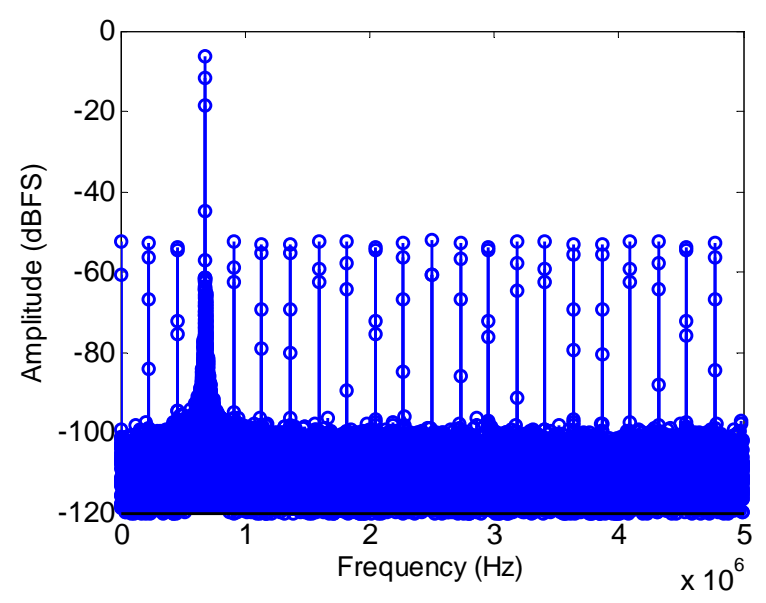

Fig.4. Simulated frequency spectrum with 20 non-overlapped higher harmonic components $f_{\text {opt }} \approx 681818.1818 \mathrm{~Hz}$

\section{Algorithm of Optimal Test Frequency DETERMINATION}

There are several frequencies $f_{\text {optl }}$ which pass (1) and which can be used as the test signal frequencies in order to achieve maximum quantity of phases in the time domain.

To use both sine fit and DFT dynamic test methods, it is necessary to find an optimal frequency that maintains maximum quantity of input phases and does not have any overlapped components in the frequency domain as well.
That frequency could be found by the following algorithm:

1. Calculate series of frequencies that passes (1)

2. Calculate desired frequency that passes (6)

3. Choose one frequency from the series of frequencies calculated in the first point with minimum difference to frequency calculated from the second point, see Fig.5

4. Verify, if there is no overlap of aliased components in the first Nyquist zone

Fig.5 shows differences between a single frequency calculated by (6) and a series of frequencies calculated by (1). The small differences usually do not influence a uniform distribution of higher harmonic components in the frequency spectrum, but for many harmonic components and significant differences, it is better to verify whether there is no overlap of aliased components by simulation.

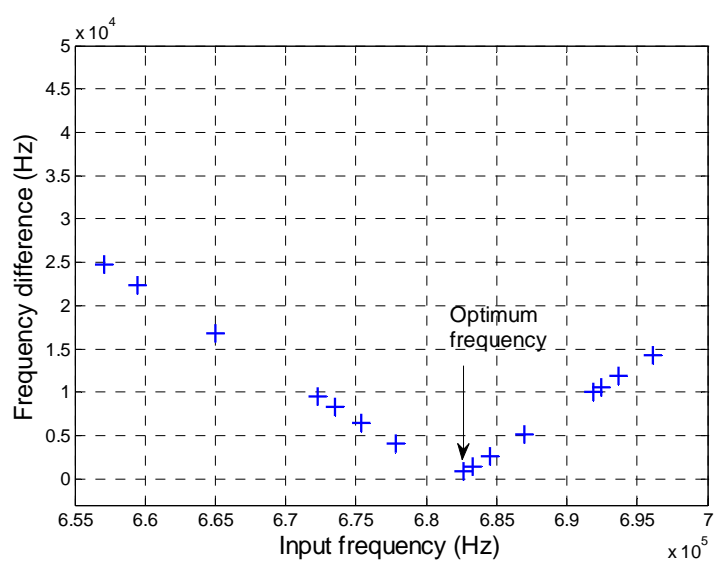

Fig.5. Frequency differences between the single frequency $f_{\text {opt2 }}$ and the series of frequencies $f_{\text {opt1 }}$

\section{EXAMPLE OF OPTIMAL FREQUENCY DETERMINATION}

As an example of optimum sine wave frequency determination, we will consider the same test setup as discussed in the previous section (sampling frequency $f_{s}=$ $10 \mathrm{MHz}$, acquired $M=32768=2^{15}$ ).

The input testing signal frequency that has maximum quantity of distinct sampled phases is given by (1). The number of acquired periods of testing signal $J$ has to be relatively prime to the number of acquired samples $M$. If $M$ is a power of two (even number), $J$ may be any odd number. More general, let $J$ be a prime number, in this case $M$ could be any number.

According to the first step of the algorithm mentioned in the previous section, it is required to find the series $f_{\text {optl }}$ of frequencies that pass (1) using the prime numbers $J$ from the range $2^{15} / 16$ to $2^{15} / 14$ which corresponds to the test signal frequency range.

The second step of the algorithm determines such test signal frequency which causes an optimal distribution of aliased higher harmonic components and prevents their overlapping. The first Nyquist zone will be divided into $(h+$ l) $=22$ intervals (distances between adjacent aliased harmonic components). The position of the fundamental harmonic component $k$ as a multiple of interval $\frac{f_{s}}{2(h+1)}$ is 
calculated by (5). The position $k$ must be an odd number, which is fulfilled for $h=21$ and $b=7$; then $k$ is equal to 3 . The optimum test signal frequency evaluated using (6) is $f_{\text {opt } 2} \approx 681818.1818 \mathrm{~Hz}$.

The test signal frequency, which should be an optimum for both mentioned criteria $f_{\text {opt }}$, should be selected from the series of frequencies $f_{\text {optl }}$ in such a way as to obtain minimum frequency difference to frequency $f_{\text {opt } 2 \text {. The }}$ minimum frequency difference $860 \mathrm{~Hz}$ is achieved with the frequency $f_{\text {opt }} \approx 682678.2227 \mathrm{~Hz}$.

The final step of the optimal test frequency determination is the verification of non-overlapping of aliased harmonic components in the first Nyquist zone. The difference between $f_{\text {opt } 2}$ and $f_{\text {opt }}$ is $860 \mathrm{~Hz}$ which causes the maximum frequency shift of the highest harmonic component equal to $22 \cdot 860=18920 \mathrm{~Hz}$. This frequency shift is insignificant compared with the distance between adjacent aliased harmonic components $\frac{f_{s}}{2(h+1)} \approx 227.272 \mathrm{kHz}$, therefore the frequency $f_{\text {opt }} \approx 682678.2227 \mathrm{~Hz}$ is a desired solution that passes all mentioned requirements. The result of measurement and simulation with test signal frequency $f_{\text {opt }}$, can be seen in Fig.6, 7.

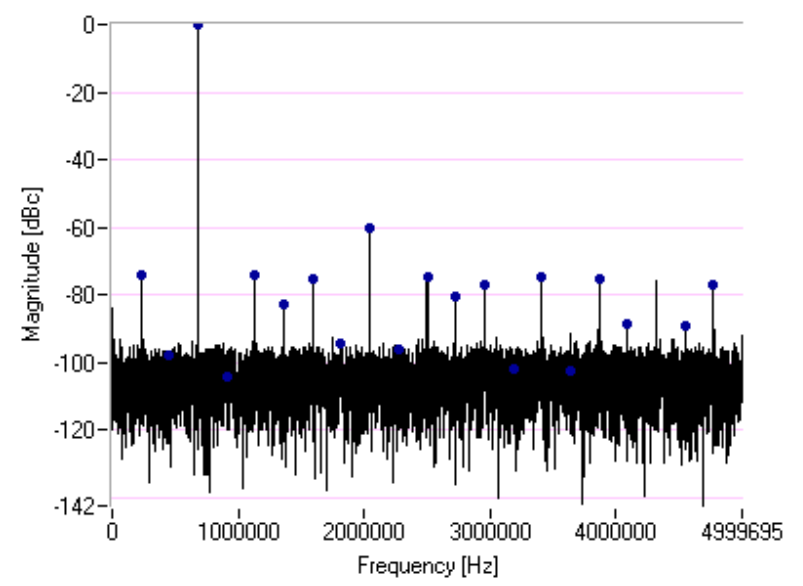

Fig.6. Measured frequency spectrum with 20 non-overlapped harmonic components $f_{\text {opt }} \approx 682678.2 \mathrm{~Hz}$

\section{CONCLUSION}

The optimum test frequency determination for the time domain and frequency domain ADC testing was discussed.

There are two requirements which have to be met. The first requirement is to get maximum quantity of distinct input phases in the acquired data stream.
The second one is to avoid overlapping of higher harmonic components aliased in the first Nyquist zone.

The proposed algorithm serves for determination of test signal frequency which is in accord with both requirements. The algorithm was verified by means of MATLAB simulations and practical measurements.

\section{ACKNOWLEDGMENT}

The research was supported by the research program No. MSM6840770015 "Research of Methods and Systems for Measurement of Physical Quantities and Measured Data Processing" of the CTU in Prague sponsored by the Ministry of Education, Youth and Sports of the Czech Republic.

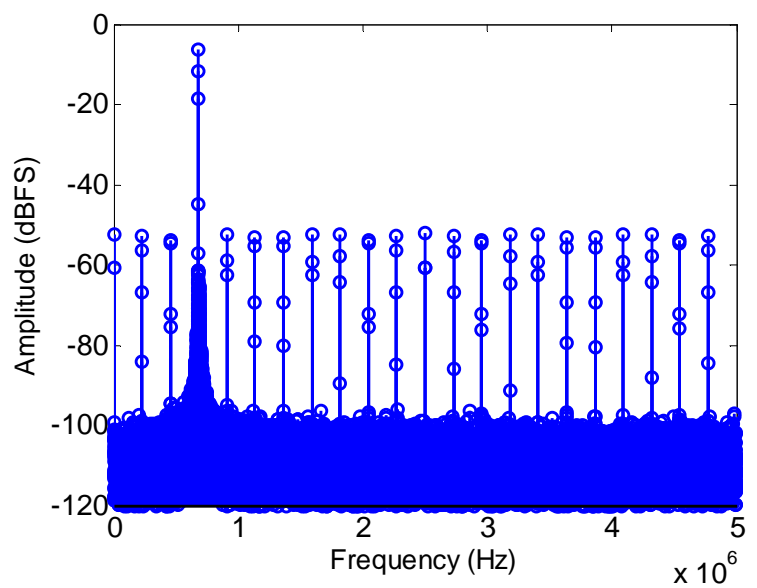

Fig. 7. Simulated frequency spectrum with 20 non-overlapped harmonic components $f_{\text {opt }} \approx 682678.2227 \mathrm{~Hz}$

\section{REFERENCES}

[1] IEEE Standards (2000). IEEE Standard for Terminology and Test Methods for Analog-to-Digital Converters. IEEE Std 1241-2000.

[2] IEEE Standards (2007). IEEE Standard for Digitizing Waveform Recorders. IEEE Std 1957-2007. (Revision of IEEE Std 1057-1994).

[3] Blair, J.J. (2005). Selecting test frequencies for sinewave tests of ADCs. IEEE Transactions On Instrumentation And Measurement, 54 (1), 73-78.

[4] Carbone, P., Petri, D. (2000). Effective frequencydomain ADC testing. IEEE Transactions On Circuits and Systems, 47 (7), 660-663.

Received November 03, 2010. Accepted December 30, 2010. 